\title{
INS-eGFP transgenic pigs: a novel reporter system for studying maturation, growth and vascularisation of neonatal islet-like cell clusters
}

\author{
Elisabeth Kemter $^{1,2}$ - Christian M. Cohrs ${ }^{3,4,5}$ - Matthias Schäfer ${ }^{6}$ Marion Schuster $^{7}$. \\ Klaus Steinmeyer ${ }^{6}$ - Lelia Wolf-van Buerck ${ }^{7}$ - Andrea Wolf ${ }^{6}$ • Annegret Wuensch ${ }^{1,2}$. \\ Mayuko Kurome $^{1,2} \cdot$ Barbara Kessler $^{1,2}$ • Valeri Zakhartchenko ${ }^{1,2} \cdot$ Matthias Loehn $^{6}$. \\ Yuri Ivashchenko ${ }^{6}$ • Jochen Seissler ${ }^{7}$ - Anke M. Schulte ${ }^{6}$ - Stephan Speier ${ }^{3,4,5}$. \\ Eckhard Wolf ${ }^{1,2,5}$
}

Received: 21 December 2016/Accepted: 24 February 2017 /Published online: 18 March 2017

(C) Springer-Verlag Berlin Heidelberg 2017

Keywords Islet maturation · Pig $\cdot$ Reporter gene

Xenotransplantation
Abbreviations
eGFP Enhanced green fluorescent protein
GSIS Glucose-stimulated insulin secretion
$\mathrm{HG} \quad$ High glucose $(15 \mathrm{mmol} / \mathrm{l})$
LG Low glucose $(3 \mathrm{mmol} / \mathrm{l})$

Dr Y. Ivashchenko, who made important contributions to this research, died in December 2016 before publication of this work.

Elisabeth Kemter

kemter@genzentrum.lmu.de

1 Chair for Molecular Animal Breeding and Biotechnology, Gene Centre, LMU Munich, Feodor-Lynen-Str. 25,

81377 Munich, Germany

2 Centre for Innovative Medical Models (CiMM), LMU Munich, Munich, Germany

3 Paul Langerhans Institute Dresden (PLID) of Helmholtz Zentrum Munich at the University Clinic Carl Gustav Carus of Technische Universität Dresden, Helmholtz Zentrum München, Neuherberg, Germany

4 DFG-Centre for Regenerative Therapies Dresden (CRTD), Faculty of Medicine, Technische Universität Dresden, Dresden, Germany

5 German Center for Diabetes Research (DZD), Munich, Neuherberg, Germany

6 Diabetes Division, Sanofi-Aventis Deutschland GmbH, Frankfurt, Germany

7 Diabetes Center-Medical Clinic Campus Innenstadt, Medizinische Klinik und Poliklinik IV, Klinikum der

Ludwig-Maximilians-Universität, LMU Munich, Munich, Germany
NICCs Neonatal islet-like cell clusters

NSG NOD.Cg-Prkdc $c^{\text {scid }} I l 2 r g^{t m l} \mathrm{Wjl} / \mathrm{SzJ}$ (NOD-scid $I l 2 r \gamma^{\text {null }}$; mice)

To the Editor: Studies of human pancreatic islet biology are limited by restricted access to high-quality human islets. Therefore, rodent islets are commonly used as model systems, although they exhibit major structural and functional differences from human islets [1]. In contrast, the spatial organisation of endocrine cells in porcine and human islets is similar [2]. Porcine islets may thus be an interesting model for human islet biology. Furthermore, islets from adult pigs and porcine neonatal islet-like cell clusters (NICCs) are a potential source for xenotransplantation to restore beta cells in patients with type 1 diabetes [3]. To facilitate the identification and isolation of porcine beta cells and the monitoring of maturation and expansion of porcine NICCs in vivo, we generated transgenic pigs that express enhanced green fluorescent protein (eGFP) under the control of the porcine insulin gene (INS) promoter (INS-eGFP transgenic pigs). The function of INS-eGFP transgenic NICCs was demonstrated by glucose-stimulated insulin secretion (GSIS) assays in vitro, and by analysis of glucose control restoration in vivo, after intramuscular transplantation of NICCs into diabetic mice. Furthermore, maturation, expansion and vascularisation of INS-eGFP transgenic NICCs were longitudinally monitored after transplantation into the anterior chamber of the mouse eye. All animal experiments were conducted with the approval and in accordance with the local animal welfare committee. Experiments were not randomised or blinded and no data was excluded. Antibody specificity was tested by immunohistochemical staining of positive and negative tissue controls. 
To generate INS-eGFP transgenic pigs, the eGFP coding sequence was cloned into a beta cell-specific expression vector with a porcine INS gene promoter sequence [4] (Fig. 1a). Porcine kidney cells were transfected, pools of stable transfected cell clones were used for somatic cell nuclear transfer, and cloned embryos were laparoscopically transferred to recipient gilts [5]. Genotyping of offspring was performed by PCR using the following primers: INS-GFP (forward) 5'-TCGTTAAGACTCTA ATGACCTC-3', (reverse) 5'-ATGAACTTCAGGGTCAGCTT GCC-3'. Transgene integration patterns were revealed by Southern blot analysis. In total, 11 INS-eGFP transgenic founder piglets with nine different integration patterns were born (Fig. 1b). Recloning of selected founder animals was performed by somatic cell nuclear transfer using kidney cells of euthanised piglets.

INS-eGFP transgenic pigs of different ages were euthanised and sections of formalin-fixed, paraffin-embedded pancreas, spleen, liver, intestine, kidney, lung, heart, adrenal gland and thyroid gland samples were analysed for eGFP expression by immunohistochemistry using rabbit polyclonal anti-GFP antibody (1:1600; no. 2555, Cell Signaling, Frankfurt am Main, Germany). eGFP was only detected in pancreatic islets (Fig. 1c, extra-pancreatic tissues not shown). Levels of eGFP fluorescence were different among founder animals; one animal presented with high eGFP, another with low eGFP, while all other animals had medium levels of eGFP fluorescence (examples are shown in Fig. 1c).

Double-immunofluorescence analysis of eGFP and insulin, glucagon or somatostatin was carried out using the following antibodies: goat polyclonal anti-GFP (12 ng/ $\mathrm{LL}$; no. ab6673, Abcam, Cambridge, UK), mouse monoclonal anti-insulin (2 ng/ $\mu \mathrm{L}$; no. 17660-16AX, USBiological, Salem, MA, USA), mouse monoclonal anti-glucagon (1:1000; no. G2654, Sigma-Aldrich, Munich, Germany) and mouse monoclonal anti-somatostatin (1:30; no. sc-74556, Santa Cruz, Dallas, TX, USA), respectively. The findings demonstrated eGFP expression exclusively in beta cells (Fig. 1d).

Using one founder with high and another one with intermediate beta cell-specific eGFP expression, two INS-eGFP transgenic lines were established and first filial generation (F1) piglets, bred in the institutional facility, were used for further characterisation of NICCs in vitro and in vivo. NICCs from <8-day-old transgenic and wild-type piglets were isolated by collagenase-V (Sigma-Aldrich) digestion of pancreas pieces [6] and cultured for 3 days in recovery medium (Ham's F12/M199 with protease inhibitors, antioxidants and additional nutrients). Full media change was carried out at day 1 and day 3 post isolation. Subsequently, NICCs were maintained in maturation medium (Ham's F10, $10 \mathrm{mmol} / 1$ glucose, $50 \mu \mathrm{mol} / 1$ 3-isobutyl-1-methylxanthine, $0.5 \%$ [wt/vol.] BSA, $2 \mathrm{mmol} / 1 \mathrm{~L}$-glutamine, $10 \mathrm{mmol} / 1 \mathrm{nico}-$ tinamide, and $1 \%$ [vol./vol.] penicillin/streptomycin stock). Half of the medium was replaced every other day.
Fig. 1 Generation and characterisation of INS-eGFP transgenic pigs. (a) Beta cell-specific eGFP expression vector with $1.3 \mathrm{~kb}$ upstream regions, exon 1 and intron 1 of the porcine INS gene, and a poly-adenylation (pA) cassette of the bovine growth hormone $(G H)$ gene, linked to a floxed neomycin resistance cassette [4]. (b) Southern blot analysis for evaluation of integration pattern and integrated copy numbers. Integration patterns of seven founder pigs are shown. Signal intensity of $10 \mathrm{pg} I N S$-eGFP plasmid (black arrow) is equivalent to one copy in $10 \mu \mathrm{g}$ genomic DNA (gDNA). gDNA was digested with the 'single cutter' restriction endonuclease enzyme EcoRI. The band representing the endogenous INS promoter is shown by the white arrow. (c) Immunohistochemical detection of eGFP expression in pancreas revealed differences in intensities among different founders. Animals were 7 days old. Chromogen staining (3,3'-diaminobenzidine [DAB]), brown colour; nuclear staining (haemalum), blue colour. Scale bars, $100 \mu \mathrm{m}$. (d) Double immunofluorescence staining of eGFP and insulin, glucagon or somatostatin (SST) showed beta cell-specific expression of eGFP. Animal was 18 months old and from line 2754. Scale bars, $50 \mu \mathrm{m}$. (e) GSIS normalised for insulin content of NICCs. GSIS of NICCs from 3-day-old piglets was tested between days 11 and 19 post isolation in a static system, where 15 hand-picked NICCs of similar size were first incubated with LG for $90 \mathrm{~min}$, followed by HG for $90 \mathrm{~min}$. GSIS assays were also performed in the presence of $100 \mathrm{nmol} / 1$ glimepiride. Insulin was quantified by porcine insulin ELISA. Data were analysed by ANOVA (Proc GLM; SAS 8.2; SAS Institute, Cary, NC, USA) taking the effects of group (transgenic vs wild-type), glucose (HG vs LG), glimepiride $(100 \mathrm{nmol} / 1$ vs $0 \mathrm{nmol} / \mathrm{l})$ and all two- and three-fold interactions into account. Insulin levels were significantly $(p<0.001)$ influenced by group, glucose and glimepiride, whereas the interactions group $\times$ glucose, group $\times$ glimepiride, and group $\times$ glucose $\times$ glimepiride were not significant. Although insulin secretion of INS-eGFP transgenic NICCs was generally higher compared with wild-type NICCs, augmentation of insulin secretion by $\mathrm{HG}$ and/or glimepiride was similar in both groups. White bars, LG; black bars, HG. Data are presented as mean \pm SEM. $n=4$ per genotype, 8 replicates each. (f) NICCs from INSeGFP transgenic (line 2904) and wild-type piglets were similarly efficient in rescuing glucose control after intramuscular transplantation into NSG mice with streptozotocin-induced diabetes. $n=6$ for wild-type transplanted NICCs; $n=3$ for transgenic transplanted NICCs. (g) At week 16 post transplantation, sections of NICC-injected muscle stained positive for insulin and GFP. Chromogen staining, DAB; nuclear staining, haemalum. Scale bars, $250 \mu \mathrm{m}$. (h) Longitudinal imaging of INS-eGFP transgenic NICCs (line 2904) in the anterior chamber of the mouse eye. Maximum intensity projections of backscatter laser light images, vessel networks and the acquired eGFP signal of the same islet engrafted on the iris of a NOD-scid mouse at the indicated time points. Histograms show quantitative data for backscatter index (an indicator of beta cell granulation, shown relative to the initial imaging time point), fractional islet vessel volume and fractional volume of eGFP-positive cells at indicated time points. Data are presented as mean \pm SEM. $n=16$ islets from six mice. Time post Tx (weeks), imaging time point in weeks after transplantation. Scale bars, $50 \mu \mathrm{m} . * p<0.05$ vs 0.5 weeks, ANOVA with Šidák correction

To evaluate if INS-eGFP reporter gene expression has a negative impact on beta cell function, GSIS of NICCs from 3-day-old transgenic and wild-type piglets was analysed simultaneously between days 11 and 19 post isolation in a static system. Pools of 15 hand-picked NICCs of similar size were first incubated in $400 \mu \mathrm{l}$ modified KRB buffer with $3 \mathrm{mmol} / \mathrm{l}$ glucose (low glucose [LG]) for $90 \mathrm{~min}$. Subsequently $100 \mu \mathrm{l}$ medium was removed and replaced by KRB with $51 \mathrm{mmol} / \mathrm{l}$ glucose, resulting in a final concentration of $15 \mathrm{mmol} / \mathrm{l}$ 
a

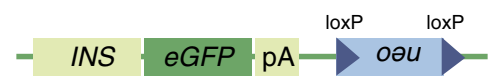

b

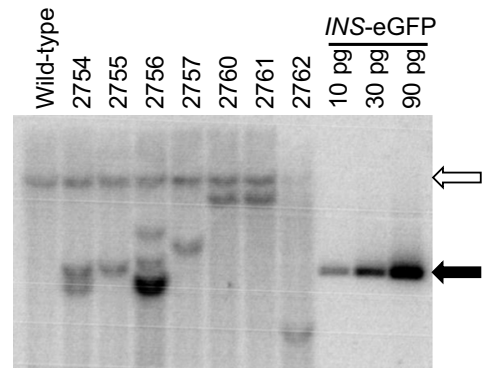

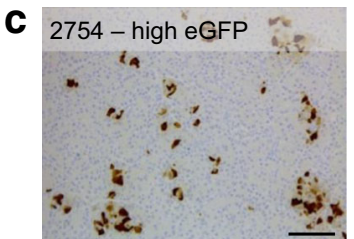

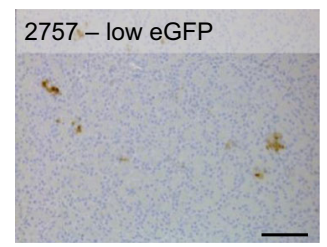

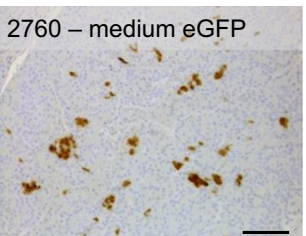

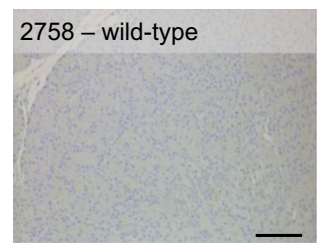

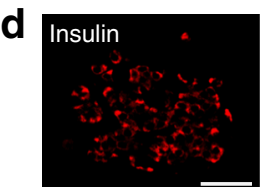
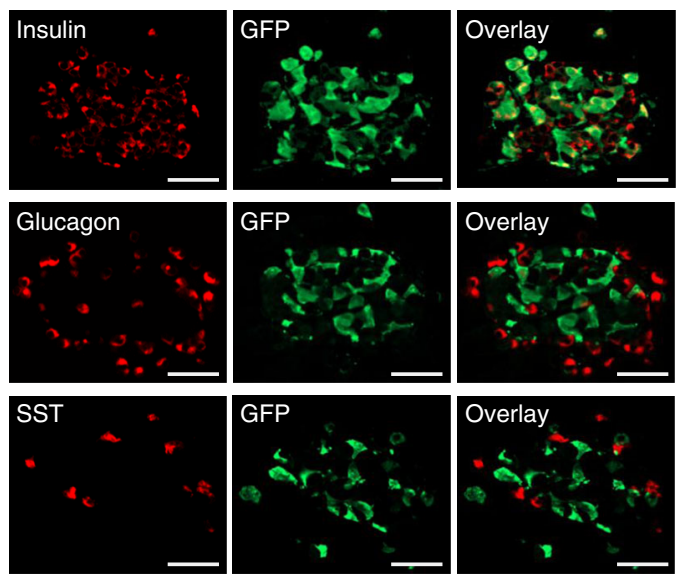

e
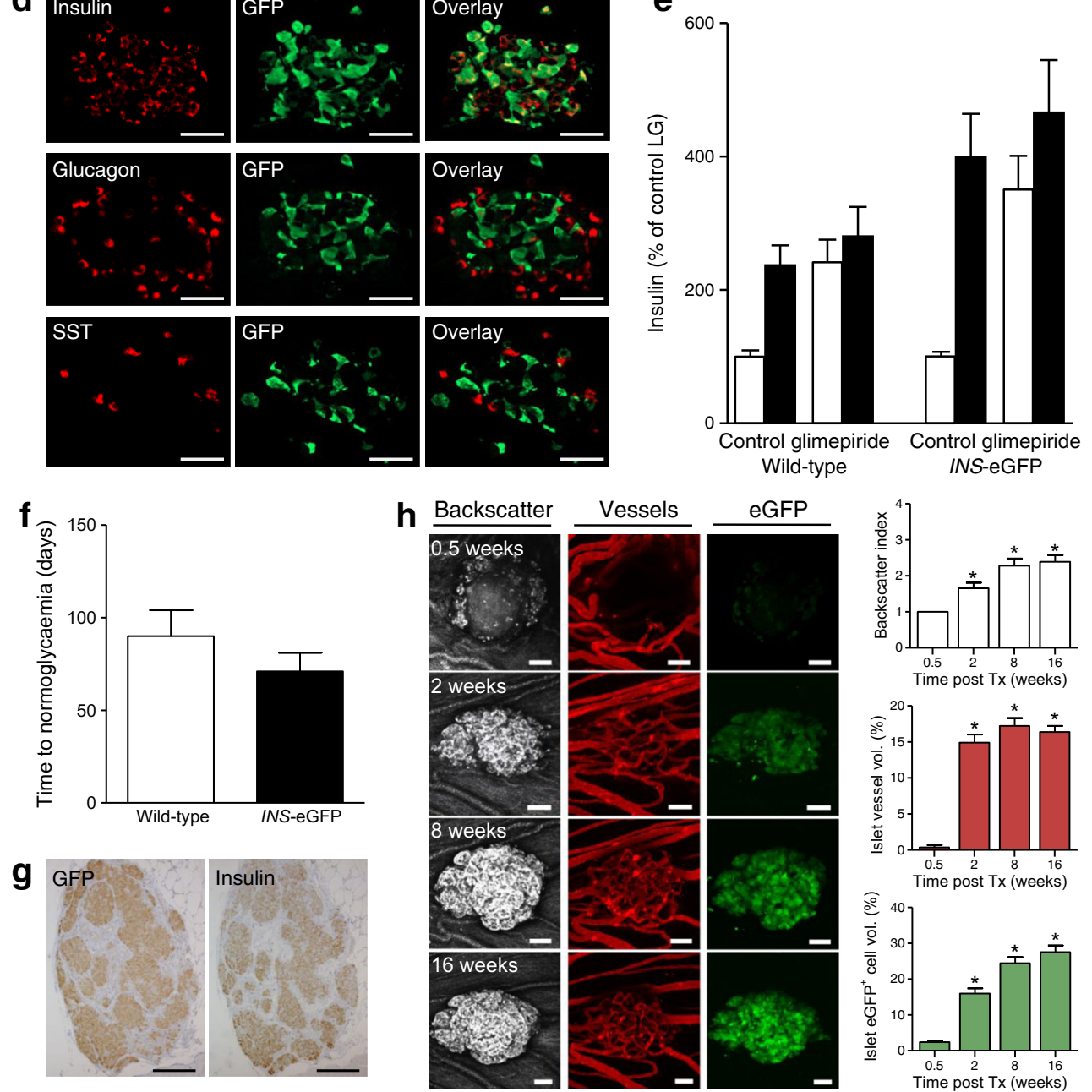

glucose (high glucose [HG]) and NICCs were incubated with HG for $90 \mathrm{~min}$. Medium was stored at $-20^{\circ} \mathrm{C}$, NICCs were resuspended in ice-cold ethanol/HCl mixture and solubilised in an ultrasonic bath (45 min). GSIS assays were also performed in the presence of $100 \mathrm{nmol} / 1$ sulfonylurea glimepiride (no. G2295, Sigma-Aldrich). Insulin in culture supernatant fractions and NICC extracts was quantified by porcine insulin ELISA (no. 10-1200-01, Mercodia, Uppsala, Sweden). Under all conditions, the ability of INS-eGFP transgenic NICCs to secrete insulin in response to glucose stimulation was equivalent or superior to that of wild-type NICCs. Baseline insulin secretion under LG conditions during 90 min was comparable between transgenic and wild-type NICCs (mean \pm SEM: $28.5 \pm 4.8 \mathrm{pg}$ and $22.1 \pm 2.7$ pg per 15 NICCs, respectively; $n=4$ per genotype, 8 replicates/donor). GSIS stimulation index (SI; calculated by insulin secretion under HG conditions/insulin secretion under LG conditions) was higher in transgenic vs wild-type NICCs (4.0 vs 2.4). In addition, a 
higher augmentation of insulin secretion by glimepiride was observed in transgenic NICCs (3.5-fold) compared with wildtype NICCs (2.4-fold; Fig. 1e).

To test the function of INS-eGFP transgenic NICCs in vivo, NICCs were transplanted into a streptozotocin-treated NOD.Cg-Prkdc ${ }^{\text {scid }} I l 2 \mathrm{rg}^{\mathrm{tm} 1 \mathrm{Wjl}} / \mathrm{SzJ}$ (abbreviated as NOD-scid $I l 2 r \gamma^{\text {null }}$; NSG) mouse model of diabetes. Experiments were conducted in healthy female and male mice, aged $>30$ and $<365$ days old, bred in the institutional facility under specificpathogen-free conditions (breeding pairs were obtained from Jackson Laboratory, Bar Harbor, ME, USA). Before transplantation, NICCs were cultured for 4-6 days in RPMI 1640 (Life Technologies [Thermo Fisher Scientific], Darmstadt, Germany) with $2 \%$ (vol./vol.) human serum albumin (Octapharm, Langenfeld, Germany), $10 \mathrm{mmol} / 1$ nicotinamide, $20 \mathrm{ng} / \mathrm{ml}$ exenatide-4 (Sigma-Aldrich) and 1\% (vol./vol.) antibioticantimycotic solution (Life Technologies). Streptozotocintreated NSG mice were injected with 2500-3000 transgenic or wild-type NICCs into the left lower hindlimb muscle [7]. Time to reach normoglycaemia was similar in mice transplanted with transgenic (median 64 days; mean $71 \pm 10$ days) or wild-type (median 72 days; mean $90 \pm 14$ days) NICCs (Fig. 1f). Immunohistological analysis was performed using a rabbit polyclonal anti-GFP antibody (1:1600; no. 2555, Cell Signaling) and a guinea pig polyclonal anti-insulin antibody (1:500; no. A0564, Dako [Agilent], Hamburg, Germany). Analysis of injected muscles at 12 and 16 weeks post transplantation revealed strong staining for insulin and, in the case of transgenic NICCs, strong staining for eGFP (Fig. 1g).

Finally, after 7 days in vitro culture, INS-eGFP transgenic NICCs were transplanted into the anterior eye chamber of 8-week-old male NOD.CB17-Prkdc ${ }^{\text {scid } / J}$ (NOD-scid) mice (Jackson Laboratory), housed in individually ventilated cages (IVCs) with access to standard rodent nutrition and water ad libitum. Mice were monitored by confocal (LSM780 NLO; Zeiss, Jena, Germany) and two-photon microscopy (Chameleon Vision II; Coherent, Santa Clara, CA, USA) at $0.5,2,8$ and 16 weeks post transplantation [8]. Backscattered laser light was detected at $633 \mathrm{~nm}$ and the backscatter index (calculated as the ratio of mean islet:iris backscatter signal from maximum intensity projections) was used as an indicator of beta cell granulation. The acquired eGFP signal was excited by a two-photon laser at $930 \mathrm{~nm}$ and was detected at 500-550 nm, while vessel networks were visualised by i.v. injection of $0.4 \mu \mathrm{mol} / 1$ Qtracker 655 (Life Technologies), with the Qtracker being excited at $930 \mathrm{~nm}$ and detected at 635-675 nm. Fractional islet vessel and eGFP-positive cell volumes were calculated using surface rendering techniques (Imaris version 8.1; Bitplane AG, Zurich, Switzerland). At 0.5 weeks after transplantation INS-eGFP transgenic NICCs showed only low granular content of beta cells, indicated by low backscatter intensity [9], and only few INS-eGFP positive cells within the clusters. Together these findings demonstrate the immature status of the NICCs. Longitudinal in vivo imaging over 16 weeks revealed an increase in granulation, INSeGFP positive fraction and islet vessel fraction, indicating how INS-eGFP transgenic NICCs mature during engraftment (Fig. 1h). After full engraftment, NICCs showed cell granulation patterns similar to adult porcine islets [10].

In conclusion, the INS-eGFP transgenic pigs generated in this study display stable eGFP expression, specifically in beta cells, and show normal islet and beta cell function in vitro and in vivo. This novel reporter system may be used for isolating porcine beta cells by fluorescence-activated cell sorting and for testing compounds on isolated beta cells or whole islets. NICCs have important advantages over adult pig islets as a source of beta cells for xenotransplantation, such as reliable isolation procedures and the ability of mass expansion [3]. However, NICCs require several weeks of in vivo maturation until they develop physiological glucose-dependent insulin secretion. NICCs expressing eGFP under the control of the INS promoter are an interesting tool for potential improvement of this maturation process, and for monitoring expansion and vascularisation of NICCs in vivo.

Acknowledgements The authors thank C. Blechinger and T. Schröter (Chair for Molecular Animal Breeding and Biotechnology, LMU Munich, Germany), as well as D. Corsiero, F. Girbig, C. Kremer, P. Kreuchauff, C. Müller, B. Meyer-Puttlitz, A. Sabel, M. Schaffer and U. Schwahn (SanofiAventis, Germany) for their excellent technical support. Some of the data were presented as an abstract at the IPITA-IXA-CTS Joint Congress meeting in Melbourne, Australia, in 2015 and at the EASD meeting in Munich, Germany, in 2016.

Data availability The datasets generated during and/or analysed during the current study are available from the corresponding author on reasonable request.

Funding This work was supported by Sanofi-Aventis Deutschland $\mathrm{GmbH}$, by the Deutsche Forschungsgemeinschaft (TRR 127), and by the German Centre for Diabetes Research (DZD). The sponsor was involved in study design and data collection and interpretation.

Duality of interest MSchäfer, KS, ML, YI and AMS are employees of Sanofi-Aventis Deutschland GmbH. AWolf is a former employee of Sanofi-Aventis Deutschland GmbH.

Contribution statement All authors made substantial contributions to conception and design, acquisition of data or analysis and interpretation of data. All authors contributed to drafting the article or revising it critically for important intellectual content and provided final approval of the version to be published. EK is the guarantor of this work.

\section{References}

1. Wang P, Fiaschi-Taesch NM, Vasavada RC, Scott DK, Garcia-Ocana A, Stewart AF (2015) Diabetes mellitus - advances and challenges in human beta-cell proliferation. Nat Rev Endocrinol 11:201-212 
2. Hoang DT, Matsunari H, Nagaya $M$ et al (2014) A conserved rule for pancreatic islet organization. PLoS One 9:e110384

3. Klymiuk N, Ludwig B, Seissler J, Reichart B, Wolf E (2016) Current concepts of using pigs as a source for beta-cell replacement therapy of type 1 diabetes. Curr Mol Bio Rep 2:73-82

4. Klymiuk N, van Buerck L, Bahr A et al (2012) Xenografted islet cell clusters from INSLEA29Y transgenic pigs rescue diabetes and prevent immune rejection in humanized mice. Diabetes 61:1527-1532

5. Kurome M, Kessler B, Wuensch A, Nagashima H, Wolf E (2015) Nuclear transfer and transgenesis in the pig. Methods Mol Biol 1222:37-59

6. Korbutt GS, Elliott JF, Ao Z, Smith DK, Warnock GL, Rajotte RV (1996) Large scale isolation, growth, and function of porcine neonatal islet cells. J Clin Invest 97:2119-2129
7. Wolf-van Buerck L, Schuster M, Baehr A et al (2015) Engraftment and reversal of diabetes after intramuscular transplantation of neonatal porcine islet-like clusters. Xenotransplantation 22:443-450

8. Chen C, Chmelova H, Cohrs CM et al (2016) Alterations in betacell calcium dynamics and efficacy outweigh islet mass adaptation in compensation of insulin resistance and prediabetes onset. Diabetes 65:2676-2685

9. Chmelova H, Cohrs CM, Chouinard JA et al (2015) Distinct roles of beta-cell mass and function during type 1 diabetes onset and remission. Diabetes 64:2148-2160

10. Cohrs CM, Chen C, Jahn SR et al (2017) Vessel network architecture of adult human islets promotes distinct cell-cell interactions in situ and is altered after transplantation. Endocrinology. doi:10. 1210/en.2016-1184 